\title{
New blazars from the cross-match of recent multi-frequency catalogs
}

\author{
A. Maselli ${ }^{1}$ - F. Massaro ${ }^{2,3}$ • R. D'Abrusco ${ }^{4}$ • \\ G. Cusumano ${ }^{1}$ - V. La Parola ${ }^{1}$ - A. Segreto ${ }^{1}$. \\ G. Tosti ${ }^{5,6}$
}

\begin{abstract}
Blazars are radio-loud active galactic nuclei well known for their non thermal emission spanning a wide range of frequencies. The Roma-BZCAT is, to date, the most comprehensive list of these sources. We performed the cross-match of several catalogs obtained from recent surveys at different frequencies to search for new blazars. We cross-matched the $1^{\text {st }}$ Swift XRT Point Source catalog with the spectroscopic sample of the $9^{\text {th }}$ Data Release of the Sloan Digital Sky Survey. Then, we performed further cross-matches with the catalogs corresponding to the Faint Images of the Radio Sky at Twenty cm survey and to the AllWISE Data re-
\end{abstract}

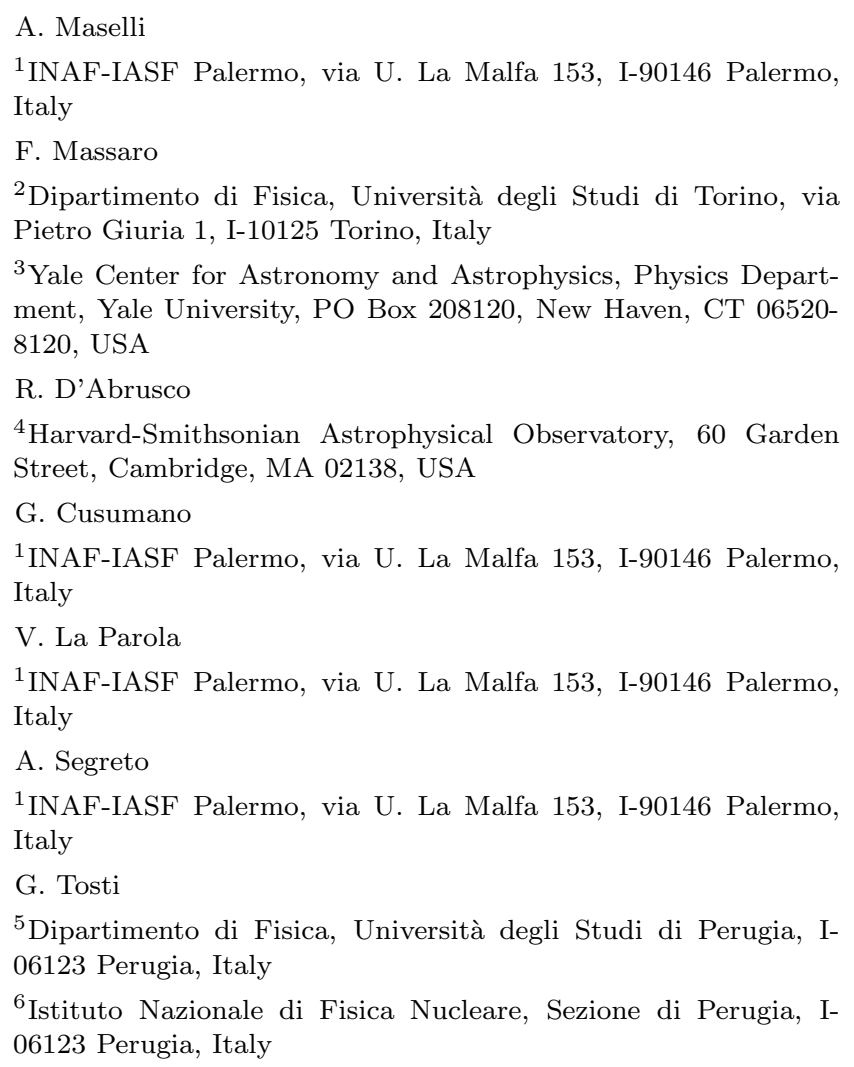

lease, focusing on sources with infrared colors similar to those of confirmed $\gamma$-ray blazars included in the Second Fermi-LAT catalog. As a result, we obtained a preliminary list of objects with all the elements needed for a proper blazar classification according to the prescriptions of the Roma-BZCAT. We carefully investigated additional properties such as their morphology and the slope of their spectral energy distribution in the radio domain, the features shown in their optical spectrum, and the luminosity in the soft $\mathrm{X}$ rays to exclude generic active galactic nuclei and focus on authentic blazar-like sources. At the end of our screening we obtained a list of 15 objects with firmly established blazar properties.

Keywords X-rays: galaxies - galaxies: active - radiation mechanisms: non-thermal.

\section{Introduction}

Blazars are peculiar radio-loud active galactic nuclei (AGNs) emitting over the whole electromagnetic spectrum, from the radio band to the $\mathrm{MeV}-\mathrm{GeV}$ range of $\gamma$ rays and extending towards the $\mathrm{TeV}$ range in the most extreme cases. Large-amplitude flux and spectral variability, on short time scales down to a few hours (i.e., Montagni et al. 2006; Gupta et al. 2008), characterize their emission. In the radio band they appear as core-dominated, flat spectrum sources. The discovery of $\gamma$-ray emission from blazars (Swanenburg et al. 1978; Hartman et al. 1999; Ackermann et al. 2011) established them as a class of extreme particle accelerators which generate some of the most energetic photons of any known extragalactic cosmic source. Within the framework of the AGN Unification Scenario (Urry and Padovani 1995), these properties are interpreted as non thermal radiation emitted by blobs of 
charged particles moving away from a central supermassive black hole at velocities close to the speed of light and in directions very close to the line of sight. In these conditions, the non thermal radiation is relativistically amplified and becomes dominant with respect to all other components of thermal origin. The blazar class includes both BL Lac objects (BL Lacs) and Flat Spectrum Radio Quasars (FSRQs), similar in the shape of their spectral energy distribution (SED) but different in their optical spectrum. BL Lacs display no or very weak emission lines with equivalent width $\mathrm{W}_{\lambda} \lesssim 5 \AA$ (Stickel et al. 1991; Stocke et al. 1991); conversely, FSRQs reveal evidence of broad emission lines. According to the Unification Scenario, BL Lacs and FSRQs are thought to be the beamed counterparts of low- (FRI) and high-luminosity (FRII) radio galaxies (Fanaroff and Riley 1974), respectively.

In recent years the number of all-sky surveys in different energy bands has considerably increased. This large amount of data is very helpful for blazar research but needs to be properly managed. At this purpose a few, well-defined and stringent criteria for blazar classification have been defined leading to the Roma-BZCAT (Massaro et al. 2009a b, 2010, 2011a). These criteria are: 1) detection in the radio band down to mJy flux densities at $1.4 \mathrm{GHz} ; 2)$ for FSRQs, a radio spectral index $\alpha_{r}$ lower than $\alpha_{r}^{\star}=0.5\left(\mathrm{~F}(\nu) \propto \nu^{-\alpha}\right)$ measured between $1.4 \mathrm{GHz}$ and $5 \mathrm{GHz}$ : this condition is not required for BL Lacs, although most of them have flat spectra; 3) compact radio morphology or, when extended, with a dominant core and a one-sided jet; 4) optical identification and analysis of the optical spectrum to establish the type of blazar; 5 ) isotropic X-ray luminosity $L_{X} \geq L_{X}^{\star}=10^{43} \mathrm{erg} \mathrm{s}^{-1}$ from a point-like source. The $4^{\text {th }}$ version of the Roma-BZCAT, available onlin£, included 3,149 objects: 1,221 BL Lacs (BZBs in the catalog, $\sim 38.8 \%$ ), 1,707 FSRQs (BZQs, $\sim 54.2 \%$ ), and 221 blazars with uncertain classification (BZUs, $\sim 7.0 \%$ ). 270 sources were included among the BL Lacs despite the lack of an optical spectrum and were therefore marked as candidates. Recent optical spectroscopic campaigns (e.g., Landoni et al. 2013; Paggi et al. 2014; Massaro et al. 2014) have been carried out to confirm the BL Lac classification of these sources; additional campaigns are ongoing.

New, complementary approaches to the blazar classification come from the evaluation of their color indices in the infrared (Massaro et al. 2011b) as well as in the optical-UV bands (Massaro et al. 2012). The discovery of peculiar infrared colors of $\gamma$-ray emitting

${ }^{1}$ http://www.asdc.asi.it/bzcat4/ blazars (D'Abrusco et al. 2012) led to the parametrization of the region that they occupy in the color space of the Wide-field Infrared Survey Explorer (WISE, Wright et al. 2010), named the WISE blazar locus (Massaro et al. 2012). This parametrization was used to develop a method (D'Abrusco et al. 2013) for the association of unidentified $\gamma$-ray sources detected by Fermi-LAT (Atwood et al. 2009) and included in the Second Fermi-LAT catalog (2FGL, Nolan et al. 2012). According to this method, the position of a generic IR source with respect to the locus can be considered a useful indicator of its blazar nature. Recently, the parametrization has been slightly modified to take into account the results of the AllWISE Data Release2 leading to the compilation of an all-sky catalog of candidate $\gamma$-ray blazars (D'Abrusco et al. 2014). This catalog includes the WISE blazar-like radio-loud sources (WIBRaLS) detected in all the four WISE filters.

In this work we provide a list of sources with firmly established blazar properties after a critical review of a preliminary list of objects obtained from the crossmatch of recent multi-frequency surveys. The paper is organised as follows: the guidelines adopted in the cross-match of the survey catalogs used in this work are illustrated in Section 2, The validation of a sample of blazars among a preliminary list of candidates is presented in Section 3 . Finally, a discussion of our results is reported in Section 4 .

The WISE magnitudes in the [3.4], [4.6], [12], and [22] $\mu \mathrm{m}$ nominal filters are expressed in the Vega system; the magnitude values of three WISE filters (namely those corresponding to [3.4], [4.6], and [12] $\mu \mathrm{m}$ ) and of the colors [3.4]-[4.6], [4.6]-[12], and [12]-[22] derived using these magnitudes, are corrected for Galactic extintion according to the extinction law presented by Draine (2003). Unless stated otherwise, we used c.g.s. units throughout and assumed a $\Lambda$ cold dark matter $(\Lambda \mathrm{CDM})$ cosmology with $\mathrm{H}_{0}=72 \mathrm{~km} \mathrm{~s}^{-1} \mathrm{Mpc}^{-1}$, $\Omega_{M}=0.26$, and $\Omega_{\Lambda}=0.74$ (Dunkley et al. 2009).

\section{Cross-match of the used survey catalogs}

We started our analysis taking into account, among the criteria for blazar's classification (Massaro et al. $2009 \mathrm{~b}$ ), those related to the point-like X-ray emission and to the possibility of analysing the optical spectrum. Therefore, we used TOPCAT 3 (Taylor 2005) to cross-match the $1^{\text {st }}$ Swift XRT Point Source cata$\log$ (1SXPS, Evans et al. 2014) with the spectroscopic

\footnotetext{
${ }^{2} \mathrm{http}$ ///wise2.ipac.caltech.edu/docs/release/allwise/expsup

${ }^{3}$ http://www.star.bris.ac.uk/ mbt/topcat/
} 
Table 1 The list of validated blazars

\begin{tabular}{|c|c|c|c|c|c|c|c|c|c|c|c|c|}
\hline name & $d_{O X}{ }^{\mathrm{a}}$ & $R_{X}^{\mathrm{b}}$ & $d_{O R}{ }^{\mathrm{c}}$ & $d_{O I}^{\mathrm{d}}$ & $S_{1.4}{ }^{\mathrm{e}}$ & $S_{5}^{\mathrm{f}}$ & $\alpha_{r}^{\mathrm{g}}$ & $r$ & $z$ & $F_{X}^{\mathrm{h}}$ & $L_{X}^{\mathrm{i}}$ & classification \\
\hline J003931.58-111102.4 & 1.2 & 4.2 & 0.41 & 0.24 & 296 & 237 & +0.18 & 19.0 & 0.553 & 6.30 & 75.1 & FSRQ \\
\hline J082438.99+405707.7 & 2.1 & 4.7 & 0.51 & 0.09 & 175 & 138 & +0.19 & 17.0 & 0.612 & 0.10 & 1.5 & FSRQ \\
\hline J082753.69+521758.3 & 2.6 & 3.8 & 0.68 & 0.14 & 181 & 292 & -0.38 & 18.9 & 0.338 & 2.50 & 9.2 & FSRQ \\
\hline J095906.96+050958.9 & 1.5 & 4.4 & 0.42 & 0.09 & 47 & 73 & -0.35 & 18.0 & 0.996 & 0.38 & 19.5 & FSRQ \\
\hline $\mathrm{J} 100033.84+132410.8$ & 1.7 & 3.9 & 0.20 & 0.13 & 53 & 34 & +0.36 & 16.7 & 1.355 & 0.74 & 82.2 & FSRQ \\
\hline $\mathrm{J} 103045.22+255522.1$ & 3.1 & 5.1 & 0.18 & 0.04 & 49 & 50 & -0.02 & 17.1 & 0.692 & 0.24 & 5.0 & FSRQ \\
\hline $\mathrm{J} 104031.62+061721.7$ & 1.1 & 3.9 & 0.12 & 0.13 & 39 & 49 & -0.18 & 19.9 & $0.743 ?$ & 0.66 & 15.9 & BL Lac \\
\hline $\mathrm{J} 110838.98+255613.2$ & 0.3 & 3.8 & 0.20 & 0.31 & 68 & 73 & -0.06 & 17.5 & 0.732 & 0.80 & 19.0 & FSRQ \\
\hline $\mathrm{J} 133631.44+031423.5$ & 5.4 & 4.3 & 0.32 & 0.75 & 62 & 111 & -0.47 & 18.9 & 1.303 & 0.12 & 12.1 & FSRQ \\
\hline $\mathrm{J} 141238.66+484447.1$ & 1.6 & 3.8 & 0.16 & 0.13 & 58 & 74 & -0.20 & 18.6 & 0.906 & 0.39 & 15.8 & FSRQ \\
\hline J142114.05+282452.8 & 3.4 & 4.1 & 0.68 & 0.03 & 49 & 40 & +0.16 & 17.8 & 0.538 & 0.46 & 5.1 & FSRQ \\
\hline $\mathrm{J} 153458.41+575625.6$ & 2.5 & 4.6 & 0.08 & 0.29 & 7 & 17 & -0.71 & 19.0 & 1.129 & 0.17 & 11.9 & FSRQ \\
\hline $\mathrm{J} 161541.21+471111.7$ & 2.9 & 4.6 & 0.23 & 0.09 & 98 & 175 & -0.46 & 17.2 & 0.199 & 1.28 & 1.4 & BL Lac \\
\hline $\mathrm{J} 162805.20+252636.8$ & 1.8 & 4.7 & 0.14 & 0.08 & 100 & 59 & +0.42 & 18.4 & 0.995 & 0.31 & 15.9 & FSRQ \\
\hline $\mathrm{J} 170634.12+361508.0$ & 4.9 & 4.4 & 0.37 & 0.14 & 19 & 27 & -0.28 & 17.9 & 0.917 & 1.06 & 44.2 & FSRQ \\
\hline
\end{tabular}

${ }^{a}$ Angular separation between the SDSS and the 1SXPS sources in arcseconds

${ }^{b} 90 \%$ error radius of the 1 SXPS source in arcseconds

${ }^{c}$ Angular separation between the SDSS source and the corresponding FIRST associated counterpart in arcseconds

${ }^{d}$ Angular separation between the SDSS source and the corresponding WISE associated counterpart in arcseconds

${ }^{e}$ Radio flux density at $1.4 \mathrm{GHz}$ in $\mathrm{mJy}$

${ }^{f}$ Radio flux density at $5 \mathrm{GHz}$ in $\mathrm{mJy}$

${ }^{g}$ Radio spectral index $\alpha_{r}$

${ }^{h}$ Unabsorbed X-ray flux $(0.3-10 \mathrm{keV})$ in $10^{-12} \mathrm{erg}^{-2} \mathrm{~s}^{-1}$

${ }^{i} \mathrm{X}$-ray luminosity $(0.3-10 \mathrm{keV})$ in $10^{44} \mathrm{erg} \mathrm{s}^{-1}$

sample of the $9^{\text {th }}$ Data Release of the Sloan Digital Sky Survey (SDSS-DR9, Ahn et al. 2012). The 1SXPS catalog resulted from the analysis of data collected by the Swift X-Ray Telescope (XRT, Burrows et al. 2005) across 8 years of operations since its launch, up to October 2012. It included 151,524 soft X-ray pointlike sources detected in the $0.3-10 \mathrm{keV}$ band with an approximately uniform sky distribution. Evans et al. (2014) carried out several checks to filter out spurious or extended sources and to express the reliability of each detected source. As a result, a quality flag which can assume three integer values (0, 1, and 2) to indicate a good, reasonable, or poor detection, respectively, was assigned to each source. In our crossmatch we considered sources from a cleaned version of the 1SXPS including only detections flagged as good or reasonable, made of 98,762 objects. We spatially cross-matched this list with the SDSS-DR9 survey catalog searching for optical counterparts, with available spectra, within the error circle (at 90\% confidence) of each X-ray source. At this purpose, we added to this $90 \%$ error radius a positional uncertainty of $2^{\prime \prime}$ to take into account the typical seeing of the SDSS. The list of 5012 SDSS sources that we obtained was then crossmatched with the WIBRaLS catalog (D'Abrusco et al. 2014). We used an overall matching radius of $3.3^{\prime \prime}$ as determined by D'Abrusco et al. (2013) in their search for the optimal value of the spatial association between blazars in the Roma-BZCAT and WISE sources, and obtained 214 objects. All the WIBRaLS have a radio counterpart. However, we kept for further investigation only objects with a radio counterpart listed in the Faint Images of the Radio sky at Twenty cm Survey (FIRST, Becker et al. 1995; White et al. 1997) since the angular resolution of this survey allows a better evaluation of the radio morphology. In the cross-match between our list of 214 SDSS sources and the latest version of the FIRST catalog (Becker et al. 2012) we adopted a matching radius of $4^{\prime \prime}$ to take into account the positional uncertainty on the FIRST source $\left(2^{\prime \prime}\right)$ in addition to the SDSS one. We obtained 201 objects including 152 blazars already present in the $4^{\text {th }}$ version of the Roma-BZCAT: consequently, we achieved a preliminary list of 49 blazar candidates whose properties were investigated in greater detail.

\section{Validation of blazar candidates}

The two main aspects that we took into account in the examination of the radio emission properties of our candidates were the morphology and the slope of their SED. Blazars are characterized by a core-dominated radio morphology; as an alternative, the additional presence of a one-sided jet can be accepted. The evidence of extended structures, and in particular those 
shaped in the form of two lobes emerging from the central core following an FRI or an FRII morphology (Fanaroff and Riley 1974) is the clear footprint of a radio galaxy instead of a blazar. Other irregular extended structures, adequately resolved by the FIRST survey, were not accepted either. As a result, we excluded from our preliminary list 11 radio galaxies, mainly characterized by a FRII morphology.

Then we estimated the slope in the SED at radio frequencies of the remaining 38 objects finding a steep spectrum for 13 of them. In our list we also found 10 objects with no measurements of flux density $S$ at radio frequencies different from $1.4 \mathrm{GHz}$; moreover, the corresponding $S_{1.4}$ values are quite low, at the level of a few mJy. The optical spectra of all these 10 sources show evident and broad emission lines typical of quasars, for which a flat radio spectrum is required (see Section 1). In these conditions the impossibility of measuring the slope of the radio spectrum prevented them from being safely classified as blazars, despite any other requirement was fulfilled.

Blazars can be distinguished from other kind of sources through the comparison of the continuum emission from the active nucleus with respect to the host galaxy contribution. A spectroscopic analysis in the optical band is able to show the mutual strength of these two components. At this purpose the Ca H\&K break absorption (Stocke et al. 1991; Marcha et al. 1996), if present, is one of the most frequently investigated features. A weak non-thermal continuum emission, reflecting in a very sharp Ca H\&K break, is the footprint of a weak central engine and probably shares little with the blazar nature, characterized by much more extreme properties. Recently, Massaro et al. (2012) have pointed out that, for objects with $z \leq 0.5$, the amount of the Ca H\&K break absorption in the SDSS spectra can be determined by computing their $(u-r)$ color index from the magnitudes of their SDSS photometry adopting the formula:

$(u-r)=(u-r)_{o b s}-0.81 A_{r}$

where $A_{r}$ is the extinction in the $r$ band. In their analysis, dominant nuclear activity in extragalactic sources has been found to correspond to $(u-r)$ values lower than a threshold $(u-r)^{\star}=1.4$. Among the 15 remaining objects only two are at redshift $z \leq 0.5$, and their $(u-r)$ color index is lower than $(u-r)^{\star}$.

The list of 15 firmly established blazars selected at the end of our investigation is shown in Table 1. Each source has been addressed using its SDSS name (column 1). The angular separation $d_{O X}$ between the SDSS and 1SXPS coordinates is reported (column 2) together with the $90 \%$ error radius $R_{X}$ of the 1SXPS

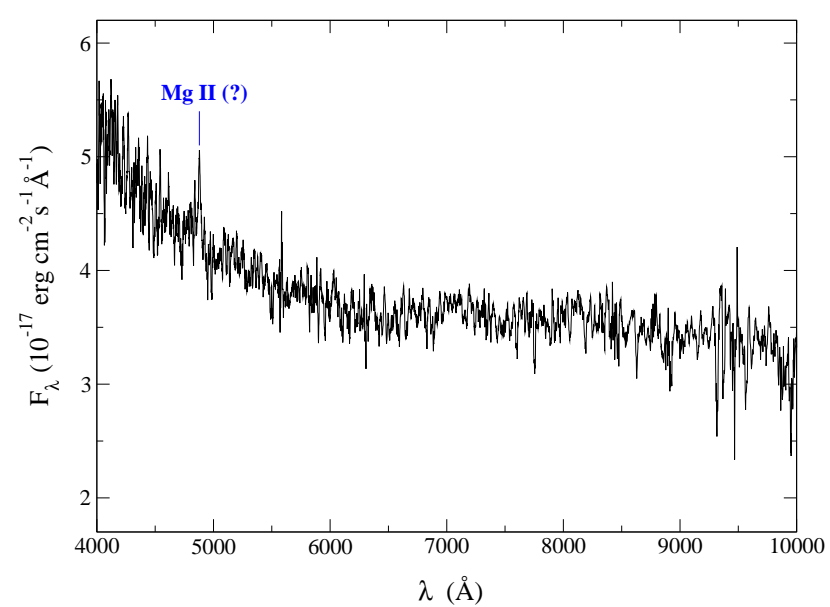

Fig. 1 The SDSS-DR9 optical spectrum of SDSS J104031.62+061721.7.

source (column 3) provided by Evans et al. (2014). We have also reported the angular separation of the SDSS source from the FIRST ( $d_{O R}$, column 4$)$ and the WISE ( $d_{O I}$, column 5) associated counterparts, respectively. The values of the radio flux density $S_{1.4}$ (column 6) have been taken from the most recent version (2012, February 16) of the FIRST survey catalog. The values $S_{5}$ (column 7 ) come from the Green Bank $6 \mathrm{~cm}$ Radio Source Catalog (GB6, Gregory et al. 1996) with the only exception of SDSS J003931.58-111102.4 for which the value found in the Parkes-MIT-NRAO survey (PMN, Wright et al. 1994) was reported. The values of the radio spectral index $\alpha_{r}$ between these two frequencies (column 8) have been calculated using the formula:

$\alpha_{r}=-1.81 \cdot \log \left(S_{5} / S_{1.4}\right)$.

The values of both the magnitude in the $r$ filter (column 9) and the redshift $z$ (column 10) come from the SDSS-DR9. One of the redshift values, corresponding to SDSS J104031.62+061721.7, is reported with a question mark as we found a flag in the optical spectrum. The flag is due to the fact that the $z$ value provided in the SDSS is based on a single, faint emission line. Assuming that this feature in the optical spectrum (Fig. 1) can be attributed to Mg II we carried out a further check on the redshift measurement. The value that we computed, which is reported in Table 1, is very similar to the one $(z=0.735)$ given in the SDSS. The values of the unabsorbed flux $F_{X}$ over the $0.3-10 \mathrm{keV}$ band (column 11) come from Evans et al. (2014). The corresponding values of the X-ray luminosity $L_{X}$ (column 12) were computed from the formula $L_{X}=4 \pi\left(d_{L}\right)^{2} \cdot F_{X}$, where the luminosity 
distance $d_{L}$ was derived from the redshift $z$ using the web calculator 4 by Wright (2006). The values that we obtained are well above the threshold $L_{X}^{\star}$ established in the Roma-BZCAT (Section 1), by at least an order of magnitude. The quality flag of all the corresponding 1SXPS sources is equal to 0 meaning a good quality X-ray detection (Section 2). Finally, in column 13 we reported the proposed classification as a BL Lac or as a FSRQ, mainly driven by the features shown in their optical spectrum.

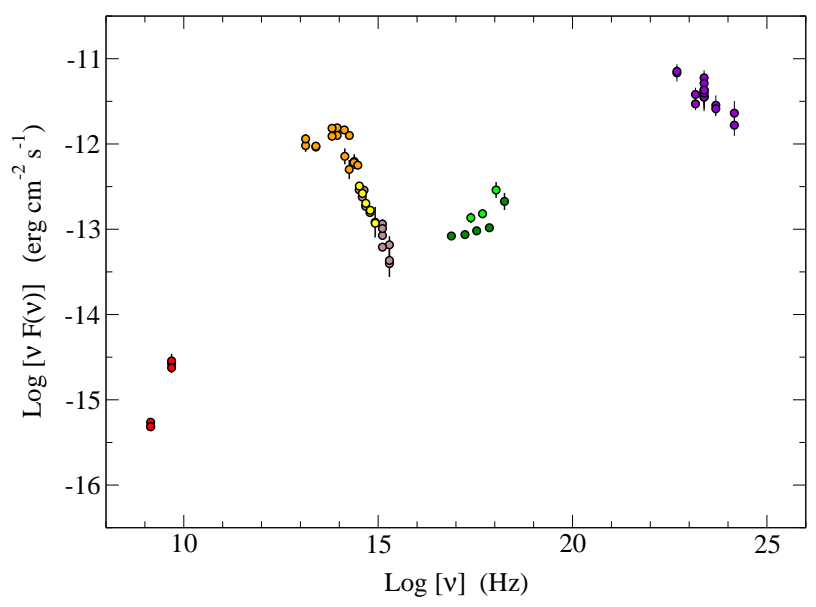

Fig. 2 The SED of SDSS J104031.62+061721.7. Red circles correspond to radio data at $1.4 \mathrm{GHz}$ and $5 \mathrm{GHz}$; orange circles correspond to infrared data from WISE, 2MASS (Skrutskie et al. 2006) and UKIDSS (Lawrence et al. 2007); SDSS data (yellow) are superposed to various data from the NED database (brown); X-ray data come from the Swift (light green) and the XMM-Newton (green) satellites; purple circles correspond to Fermi data.

\section{Discussion}

Blazars are certainly not the only sources characterized by a multifrequency emission. In recent years their peculiar properties have been well defined by restrictive criteria: the need for establishing them has become quite urgent as a consequence of the rapid increase in the lists of candidates, a large number of which is simply the result of automatic cross-matches of the various all-sky surveys obtained by spacecraft telescopes at different energy bands. For this reason, a careful check of the overall properties is highly recommended to support any candidate before validating the blazar

${ }^{4} \mathrm{http}: / /$ www.astro.ucla.edu/ wright/CosmoCalc.html classification. At the end of our screening procedure, that includes some steps that could not be carried out automatically, we obtained a list of 15 blazars. We verified that the SED of all our blazars is consistent with the characteristic double-bumped shape, the former attributed to synchrotron radiation and the latter to inverse Compton emission. One of them is reported as example in Fig. 2. All the values of the radio spectral index $\alpha_{r}$ are strictly consistent with a flat radio spectrum. According to the classification that we propose all the sources of our list, with two exceptions, are FSRQs with a mean redshift value $\bar{z}=0.851$. FSRQs are generally found among low synchrotron peak (LSP, Abdo et al. 2010) sources: in these conditions, the detection in the soft $\mathrm{X}$ rays corresponds to the inverse Compton emission.

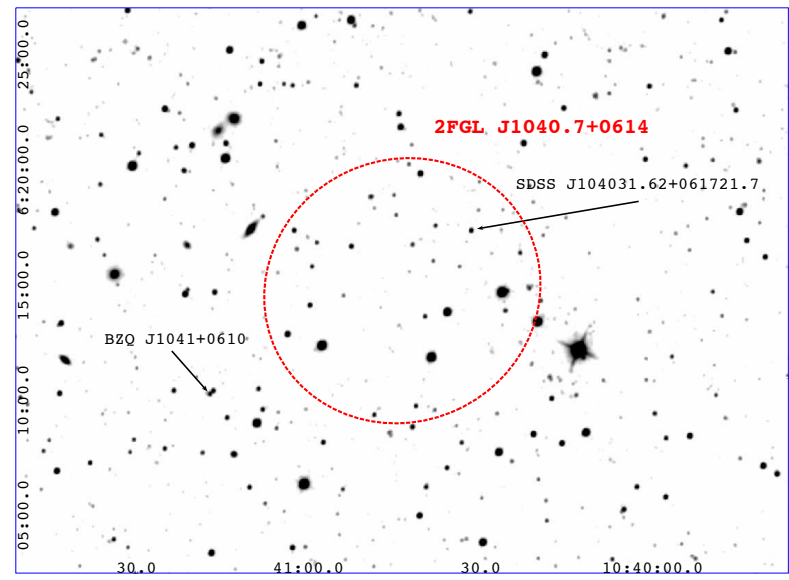

Fig. 3 WISE $3.4 \mu \mathrm{m}$ sky map centered at the position of 2FGL J1040.7+0614. The BL Lac object found by our procedure, SDSS J104031.62+061721.7, is within the error region of the $\gamma$-ray source (red crossed line) at variance with BZQ J1041+0610, the counterpart associated in the 2LAC.

In the optical spectrum of SDSS J104031.62+061721.7 (Fig. 1) there is evidence of continuum emission with no broad emission lines, suggesting a BL Lac classification. Considerable variability in the optical band was reported by Bauer et al. (2009). We note (Fig. 33) that this source is within the $95 \%$ error circle $\theta_{95}=$ $5.88^{\prime}$ of the $\gamma$-ray source 2FGL J1040.7+0614; the angular separation between the SDSS and the 2FGL coordinates is $3.96^{\prime}$. Conversely, the counterpart $4 \mathrm{C}+06.41$ (named BZQ J1041+0610 in the Roma-BZCAT) currently associated to this $\gamma$-ray source in the Second LAT 
AGN Catalog (2LAC, Ackermann et al. 2011) is outside of the 2FGL error circle, at a distance of $9.45^{\prime}$; for this reason, their association is given with a low bayesian probability. Therefore, we consider the eventuality that a contribution to the $\gamma$-ray emission corresponding to 2FGL J1040.7+0614 might be attributed to SDSS J104031.62+061721.7, possibly in addition to BZQ J1041+0610.

The other source that does not show broad emission lines in its optical spectrum is SDSS J161541.21+471111.7. The value that we found for its color index $(u-$ $r)=1.34$, even if lower than the threshold $(u-r)^{\star}$ established by Massaro et al. (2012), suggests a relatively weak non-thermal contribution with respect to the host galaxy. Moreover, this source was recently addressed by Massaro et al. (2013) as a possible counterpart of the unidentified $\gamma$-ray source (UGS) 2FGL J1614.8+4703 detected by Fermi-LAT.

If we consider the totality of blazars selected by our method, including the 152 already reported in the $4^{\text {th }}$ version of the Roma-BZCAT, in our list of $167 \mathrm{ob}-$ jects we found 72 BZBs $(\sim 43.1 \%), 85$ BZQs $(\sim 50.9 \%)$ and 10 BZUs $(\sim 6.0 \%)$, respectively. These rates are in agreement with those corresponding to the whole blazar population as reported in the Roma-BZCAT (Section 1).

\section{Acknowledgements}

The work is supported by the ASI grant I/004/11/0 and by the NASA grants NNX12AO97G and NNX13AP20G. R. DAbrusco acknowledges the SI competitive research grant "A New Astro-Archeology Probe of the Merging Evolution of Galaxies" for support. R. DAbrusco's work was also partially supported by the Chandra Xray Center (CXC), which is operated by the Smithsonian Astrophysical Observatory (SAO) under NASA contract NAS8-03060. The work by G. Tosti is supported by the ASI/INAF contract I/005/12/0. TOPCAT and SAOImage DS9 were used extensively in this work for the preparation and manipulation of the tabular data and the images. This research has made use of data obtained from the High Energy Astrophysics Science Archive Research Center (HEASARC) provided by NASA's Goddard Space Flight Center; the SIMBAD database operated at CDS, Strasbourg, France; the NASA/IPAC Extragalactic Database (NED) operated by the Jet Propulsion Laboratory, California Institute of Technology, under contract with the National Aeronautics and Space Administration. Part of this work is based on archival data, software or on-line services provided by the ASI Science Data Center. This publication makes use of data products from the Widefield Infrared Survey Explorer, which is a joint project of the University of California, Los Angeles, and the Jet Propulsion Laboratory/California Institute of Technology, funded by the National Aeronautics and Space Administration. This work made use of data supplied by the UK Swift Science Data Centre at the University of Leicester. Funding for SDSS-III has been provided by the Alfred P. Sloan Foundation, the Participating Institutions, the National Science Foundation, and the U.S. Department of Energy Office of Science. The SDSS-III web site is http://www.sdss3.org/. SDSS-III is managed by the Astrophysical Research Consortium for the Participating Institutions of the SDSS-III Collaboration including the University of Arizona, the Brazilian Participation Group, Brookhaven National Laboratory, University of Cambridge, Carnegie Mellon University, University of Florida, the French Participation Group, the German Participation Group, Harvard University, the Instituto de Astrofisica de Canarias, the Michigan State/Notre Dame/JINA Participation Group, Johns Hopkins University, Lawrence Berkeley National Laboratory, Max Planck Institute for Astrophysics, Max Planck Institute for Extraterrestrial Physics, New Mexico State University, New York University, Ohio State University, Pennsylvania State University, University of Portsmouth, Princeton University, the Spanish Participation Group, University of Tokyo, University of Utah, Vanderbilt University, University of Virginia, University of Washington, and Yale University. 


\section{References}

Abdo, A.A., Ackermann, M., Agudo, I., Ajello, M., Aller, H.D., Aller, M.F., Angelakis, E., Arkharov, A.A., Axelsson, M., Bach, U., et al.: Astrophys. J. 716, 30 (2010). 0912.2040. doi:10.1088/0004-637X/716/1/30

Ackermann, M., Ajello, M., Allafort, A., Antolini, E., Atwood, W.B., Axelsson, M., Baldini, L., Ballet, J., Barbiellini, G., Bastieri, D., Bechtol, K., Bellazzini, R., Berenji, B., Blandford, R.D., Bloom, E.D., Bonamente, E., Borgland, A.W., Bottacini, E., Bouvier, A., Bregeon, J., Brigida, M., Bruel, P., Buehler, R., Burnett, T.H., Buson, S., Caliandro, G.A., Cameron, R.A., Caraveo, P.A., Casandjian, J.M., Cavazzuti, E., Cecchi, C., Charles, E., Cheung, C.C., Chiang, J., Ciprini, S., Claus, R., CohenTanugi, J., Conrad, J., Costamante, L., Cutini, S., de Angelis, A., de Palma, F., Dermer, C.D., Digel, S.W., Silva, E.d.C.e., Drell, P.S., Dubois, R., Escande, L., Favuzzi, C., Fegan, S.J., Ferrara, E.C., Finke, J., Focke, W.B., Fortin, P., Frailis, M., Fukazawa, Y., Funk, S., Fusco, P., Gargano, F., Gasparrini, D., Gehrels, N., Germani, S., Giebels, B., Giglietto, N., Giommi, P., Giordano, F., Giroletti, M., Glanzman, T., Godfrey, G., Grenier, I.A., Grove, J.E., Guiriec, S., Gustafsson, M., Hadasch, D., Hayashida, M., Hays, E., Healey, S.E., Horan, D., Hou, X., Hughes, R.E., Iafrate, G., Jóhannesson, G., Johnson, A.S., Johnson, W.N., Kamae, T., Katagiri, H., Kataoka, J., Knödlseder, J., Kuss, M., Lande, J., Larsson, S., Latronico, L., Longo, F., Loparco, F., Lott, B., Lovellette, M.N., Lubrano, P., Madejski, G.M., Mazziotta, M.N., McConville, W., McEnery, J.E., Michelson, P.F., Mitthumsiri, W., Mizuno, T., Moiseev, A.A., Monte, C., Monzani, M.E., Moretti, E., Morselli, A., Moskalenko, I.V., Murgia, S., Nakamori, T., NaumannGodo, M., Nolan, P.L., Norris, J.P., Nuss, E., Ohno, M., Ohsugi, T., Okumura, A., Omodei, N., Orienti, M., Orlando, E., Ormes, J.F., Ozaki, M., Paneque, D., Parent, D., Pesce-Rollins, M., Pierbattista, M., Piranomonte, S., Piron, F., Pivato, G., Porter, T.A., Rainò, S., Rando, R., Razzano, M., Razzaque, S., Reimer, A., Reimer, O., Ritz, S., Rochester, L.S., Romani, R.W., Roth, M., Sanchez, D.A., Sbarra, C., Scargle, J.D., Schalk, T.L., Sgrò, C., Shaw, M.S., Siskind, E.J., Spandre, G., Spinelli, P., Strong, A.W., Suson, D.J., Tajima, H., Takahashi, H., Takahashi, T., Tanaka, T., Thayer, J.G., Thayer, J.B., Thompson, D.J., Tibaldo, L., Tinivella, M., Torres, D.F., Tosti, G., Troja, E., Uchiyama, Y., Vandenbroucke, J., Vasileiou, V., Vianello, G., Vitale, V., Waite, A.P., Wallace, E., Wang, P., Winer, B.L., Wood, D.L., Wood, K.S., Zimmer, S.: Astrophys. J. 743, 171 (2011). 1108.1420. doi:10.1088/0004-637X/743/2/171

Ahn, C.P., Alexandroff, R., Allende Prieto, C., Anderson, S.F., Anderton, T., Andrews, B.H., Aubourg, É., Bailey, S., Balbinot, E., Barnes, R., et al.: Astrophys. J. Suppl. Ser. 203, 21 (2012). 1207.7137. doi:10.1088/00670049/203/2/21

Atwood, W.B., Abdo, A.A., Ackermann, M., Althouse, W., Anderson, B., Axelsson, M., Baldini, L., Ballet, J., Band, D.L., Barbiellini, G., et al.: Astrophys. J. 697, 1071 (2009). 0902.1089. doi:10.1088/0004-637X/697/2/1071
Bauer, A., Baltay, C., Coppi, P., Donalek, C., Drake, A., Djorgovski, S.G., Ellman, N., Glikman, E., Graham, M., Jerke, J., Mahabal, A., Rabinowitz, D., Scalzo, R., Williams, R.: Astrophys. J. 705, 46 (2009). 0909.0014. doi:10.1088/0004-637X/705/1/46

Becker, R.H., White, R.L., Helfand, D.J.: Astrophys. J. 450, 559 (1995). doi:10.1086/176166

Becker, R.H., Helfand, D.J., White, R.L., Gregg, M.D., Laurent-Muehlheisen, S.A.: VizieR Online Data Catalog 8090, 0 (2012)

Burrows, D.N., Hill, J.E., Nousek, J.A., Kennea, J.A., Wells, A., Osborne, J.P., Abbey, A.F., Beardmore, A., Mukerjee, K., Short, A.D.T., Chincarini, G., Campana, S., Citterio, O., Moretti, A., Pagani, C., Tagliaferri, G., Giommi, P., Capalbi, M., Tamburelli, F., Angelini, L., Cusumano, G., Bräuninger, H.W., Burkert, W., Hartner, G.D.: Space Sci. Rev. 120, 165 (2005). astro-ph/0508071. doi:10.1007/s11214-005-5097-2

D'Abrusco, R., Massaro, F., Ajello, M., Grindlay, J.E., Smith, H.A., Tosti, G.: Astrophys. J. 748, 68 (2012). 1203.0568. doi:10.1088/0004-637X/748/1/68

D'Abrusco, R., Massaro, F., Paggi, A., Masetti, N., Tosti, G., Giroletti, M., Smith, H.A.: Astrophys. J. Suppl. Ser. 206, 12 (2013). 1303.3002. doi:10.1088/00670049/206/2/12

D'Abrusco, R., Massaro, F., Paggi, A., Smith, H.A., Masetti, N., Landoni, M., Tosti, G.: Astrophys. J. Suppl. Ser. 215, 14 (2014). 1410.0029. doi:10.1088/00670049/215/1/14

Draine, B.T.: Annu. Rev. Astron. Astrophys. 41, 241 (2003). astro-ph/0304489. doi:10.1146/annurev.astro.41.011802.094840

Dunkley, J., Komatsu, E., Nolta, M.R., Spergel, D.N., Larson, D., Hinshaw, G., Page, L., Bennett, C.L., Gold, B., Jarosik, N., Weiland, J.L., Halpern, M., Hill, R.S., Kogut, A., Limon, M., Meyer, S.S., Tucker, G.S., Wollack, E., Wright, E.L.: Astrophys. J. Suppl. Ser. 180, 306 (2009). 0803.0586. doi:10.1088/0067-0049/180/2/306

Evans, P.A., Osborne, J.P., Beardmore, A.P., Page, K.L., Willingale, R., Mountford, C.J., Pagani, C., Burrows, D.N., Kennea, J.A., Perri, M., Tagliaferri, G., Gehrels, N.: Astrophys. J. Suppl. Ser. 210, 8 (2014). 1311.5368. doi:10.1088/0067-0049/210/1/8

Fanaroff, B.L., Riley, J.M.: Mon. Not. R. Astron. Soc. 167, $31(1974)$

Gregory, P.C., Scott, W.K., Douglas, K., Condon, J.J.: Astrophys. J. Suppl. Ser. 103, 427 (1996). doi:10.1086/192282

Gupta, A.C., Fan, J.H., Bai, J.M., Wagner, S.J.: Astron. J. 135, 1384 (2008). 0801.1217. doi:10.1088/0004$6256 / 135 / 4 / 1384$

Hartman, R.C., Bertsch, D.L., Bloom, S.D., Chen, A.W., Deines-Jones, P., Esposito, J.A., Fichtel, C.E., Friedlander, D.P., Hunter, S.D., McDonald, L.M., Sreekumar, P., Thompson, D.J., Jones, B.B., Lin, Y.C., Michelson, P.F., Nolan, P.L., Tompkins, W.F., Kanbach, G., MayerHasselwander, H.A., Mücke, A., Pohl, M., Reimer, O., Kniffen, D.A., Schneid, E.J., von Montigny, C., Mukherjee, R., Dingus, B.L.: Astrophys. J. Suppl. Ser. 123, 79 (1999). doi:10.1086/313231 
Landoni, M., Falomo, R., Treves, A., Sbarufatti, B., Barattini, M., Decarli, R., Kotilainen, J.: Astron. J. 145, 114 (2013). 1302.2591. doi:10.1088/0004-6256/145/4/114

Lawrence, A., Warren, S.J., Almaini, O., Edge, A.C., Hambly, N.C., Jameson, R.F., Lucas, P., Casali, M., Adamson, A., Dye, S., Emerson, J.P., Foucaud, S., Hewett, P., Hirst, P., Hodgkin, S.T., Irwin, M.J., Lodieu, N., McMahon, R.G., Simpson, C., Smail, I., Mortlock, D., Folger, M.: Mon. Not. R. Astron. Soc. 379, 1599 (2007). astroph/0604426. doi:10.1111/j.1365-2966.2007.12040.x

Marcha, M.J.M., Browne, I.W.A., Impey, C.D., Smith, P.S.: Mon. Not. R. Astron. Soc. 281, 425 (1996)

Massaro, E., Nesci, R., Piranomonte, S.: Mon. Not. R. Astron. Soc. 422, 2322 (2012). 1202.4614. doi:10.1111/j.1365 2966.2012.20782.x

Massaro, E., Giommi, P., Leto, C., Marchegiani, P., Maselli, A., Perri, M., Piranomonte, S., Sclavi, S.: VizieR Online Data Catalog 349, 50691 (2009a)

Massaro, E., Giommi, P., Leto, C., Marchegiani, P., Maselli, A., Perri, M., Piranomonte, S., Sclavi, S.: Astron. Astrophys. 495, 691 (2009b). 0810.2206. doi:10.1051/00046361:200810161

Massaro, E., Giommi, P., Leto, C., Marchegiani, P., Maselli, A., Perri, M., Piranomonte, S., Sclavi, S.: ArXiv e-prints (2010). 1006.0922

Massaro, E., Giommi, P., Leto, C., Marchegiani, P., Maselli, A., Perri, M., Piranomonte, S.: Multifrequency Catalogue of Blazars (3rd Edition). ARACNE Editrice, Rome, Italy, 118 pages, ??? (2011a)

Massaro, F., D'Abrusco, R., Ajello, M., Grindlay, J.E., Smith, H.A.: Astrophys. J. Lett. 740, 48 (2011b). 1203.0304. doi:10.1088/2041-8205/740/2/L48

Massaro, F., D'Abrusco, R., Tosti, G., Ajello, M., Gasparrini, D., Grindlay, J.E., Smith, H.A.: Astrophys. J. 750, 138 (2012). 1203.1330. doi:10.1088/0004-637X/750/2/138

Massaro, F., D'Abrusco, R., Paggi, A., Masetti, N., Giroletti, M., Tosti, G., Smith, H.A., Funk, S.: Astrophys. J. Suppl. Ser. 206, 13 (2013). 1303.3585. doi:10.1088/00670049/206/2/13

Massaro, F., Masetti, N., D’Abrusco, R., Paggi, A., Funk, S.: Astron. J. 148, 66 (2014). doi:10.1088/0004$6256 / 148 / 4 / 66$

Montagni, F., Maselli, A., Massaro, E., Nesci, R., Sclavi, S., Maesano, M.: Astron. Astrophys. 451, 435 (2006). astro-ph/0512471. doi:10.1051/0004-6361:20053874

Nolan, P.L., Abdo, A.A., Ackermann, M., Ajello, M., Allafort, A., Antolini, E., Atwood, W.B., Axelsson, M., Baldini, L., Ballet, J., et al.: Astrophys. J. Suppl. Ser. 199, 31 (2012). 1108.1435. doi:10.1088/0067-0049/199/2/31

Paggi, A., Milisavljevic, D., Masetti, N., Jiménez-Bailón, E., Chavushyan, V., D'Abrusco, R., Massaro, F., Giroletti, M., Smith, H.A., Margutti, R., Tosti, G., MartínezGalarza, J.R., Otí-Floranes, H., Landoni, M., Grindlay, J.E., Funk, S.: Astron. J. 147, 112 (2014). 1404.5211. doi:10.1088/0004-6256/147/5/112

Skrutskie, M.F., Cutri, R.M., Stiening, R., Weinberg, M.D., Schneider, S., Carpenter, J.M., Beichman, C., Capps, R., Chester, T., Elias, J., Huchra, J., Liebert, J., Lonsdale, C., Monet, D.G., Price, S., Seitzer, P., Jarrett, T., Kirkpatrick, J.D., Gizis, J.E., Howard, E., Evans, T., Fowler,
J., Fullmer, L., Hurt, R., Light, R., Kopan, E.L., Marsh, K.A., McCallon, H.L., Tam, R., Van Dyk, S., Wheelock, S.: Astron. J. 131, 1163 (2006). doi:10.1086/498708

Stickel, M., Padovani, P., Urry, C.M., Fried, J.W., Kuehr, H.: Astrophys. J. 374, 431 (1991). doi:10.1086/170133

Stocke, J.T., Morris, S.L., Gioia, I.M., Maccacaro, T., Schild, R., Wolter, A., Fleming, T.A., Henry, J.P.: Astrophys. J. Suppl. Ser. 76, 813 (1991). doi:10.1086/191582

Swanenburg, B.N., Hermsen, W., Bennett, K., Bignami, G.F., Caraveo, P., Kanbach, G., Mayer-Hasselwander, H.A., Masnou, J.L., Paul, J.A., Sacco, B.: Nature 275, 298 (1978). doi:10.1038/275298a0

Taylor, M.B.: In: Shopbell, P., Britton, M., Ebert, R. (eds.) Astronomical Data Analysis Software and Systems XIV. Astronomical Society of the Pacific Conference Series, vol. 347, p. 29 (2005)

Urry, C.M., Padovani, P.: Publ. Astron. Soc. Pac. 107, 803 (1995). astro-ph/9506063. doi:10.1086/133630

White, R.L., Becker, R.H., Helfand, D.J., Gregg, M.D.: Astrophys. J. 475, 479 (1997)

Wright, A.E., Griffith, M.R., Burke, B.F., Ekers, R.D.: Astrophys. J. Suppl. Ser. 91, 111 (1994). doi:10.1086/191939

Wright, E.L.: Publ. Astron. Soc. Pac. 118, 1711 (2006). astro-ph/0609593. doi:10.1086/510102

Wright, E.L., Eisenhardt, P.R.M., Mainzer, A.K., Ressler, M.E., Cutri, R.M., Jarrett, T., Kirkpatrick, J.D., Padgett, D., McMillan, R.S., Skrutskie, M., Stanford, S.A., Cohen, M., Walker, R.G., Mather, J.C., Leisawitz, D., Gautier, T.N. III, McLean, I., Benford, D., Lonsdale, C.J., Blain, A., Mendez, B., Irace, W.R., Duval, V., Liu, F., Royer, D., Heinrichsen, I., Howard, J., Shannon, M., Kendall, M., Walsh, A.L., Larsen, M., Cardon, J.G., Schick, S., Schwalm, M., Abid, M., Fabinsky, B., Naes, L., Tsai, C.-W.: Astron. J. 140, 1868 (2010). 1008.0031. doi:10.1088/0004-6256/140/6/1868

This manuscript was prepared with the AAS LATEX macros v5.2. 